\title{
Malignant Proliferating Pilar Tumor
}

National Cancer Institute

\section{Source}

National Cancer Institute. Malignant Proliferating Pilar Tumor. NCI Thesaurus. Code C43327.

It is a rare malignancy that arises from the outer root sheath of the hair follicle. It usually occurs in the scalp, face and trunk. Histopathology shows cords of malignant squamous cells infiltrating into the dermis. 